\title{
Improving access to clinical guidance: redevelopment of a junior doctor intranet page
}

Graham Walkden, Sinead Millwood, Andrew Cavanagh

North Bristol NHS Trust, United Kingdom

\begin{abstract}
Junior doctors frequently rely on electronic access to clinical guidelines to inform assessment and management, particularly whilst on-call and occasionally during emergencies. Difficulties in locating and accessing up to date guidance from different hospital intranet sites can lead to delays or errors in patient management. We used a focus group and email feedback to redesign an intranet site for junior doctors which logically organised the documents which doctors said they needed access to in one readily accessible location. A quality improvement project was carried out over six months, testing two iterations of the new junior doctors' intranet site before a third version was launched and evaluated. Their performance was measured by the number of mouse clicks and the time required for doctors to find a representative subset of five guidelines, and revisions were made at each cycle based on feedback from doctors and stakeholders. Cumulatively, we demonstrated a decrease in the total number of clicks required to access the sample of guidelines from 18 to 12 clicks, a corresponding decrease in the time required to access the sample of guidelines from 130 seconds to 22 seconds, and an increase in user satisfaction. We maintained one-click access to emergency guidance. In conclusion, we have developed and implemented an electronic resource for junior doctors which provides more immediate access to both emergency and non-emergency clinical guidance. To ensure the resource remains up to date, it will be maintained by Foundation Programme representatives at our hospital on a rolling basis.
\end{abstract}

\section{Problem}

Junior doctors in hospitals thoughout the United Kingdom rely on electronic access to clinical guidance and other documents to inform clinical assessment and management. Previous work at our centre that was published in 2014 showed, for example, that $48 \%$ of junior doctors who were surveyed at baseline were unable to locate upper gastrointestinal bleed guidelines within five minutes [1]. Difficulties in locating and accessing up to date guidance from hospital intranet may lead to delays and errors in patient management and could potentially contribute to adverse outcomes. Since inception, the junior doctors' intranet page at North Bristol NHS Trust (Bristol, United Kingdom) had been populated with a large number of additional documents, all presented on a single page, making it disorganised and difficult to navigate. Since it had not been updated since launch, it did not list all of the documents that doctors needed access to. It was therefore not fit for purpose.

\section{Background}

The original junior doctors' guidelines intranet page was designed to give junior doctors quick access to the emergency clinical guidelines that may be needed on-call. However, the single page had evolved to include many additional guidelines and documents which were becoming difficult to find as they were not presented in any particular order. The hospital intranet has a number of different sites in which relevant and useful guidelines can be accessed, and it is only after months of working in a job that junior doctors said that they were able to find the guidelines they need.

The project aims to create a Junior Doctor Guidelines page which:
1. Logically indexes all the guidelines a junior doctor might need,

2. Has one-click access to emergency guidance, and

3 . Is easily navigable for users.

\section{Baseline measurement}

1. We emailed all Foundation doctors at our hospital, asking for feedback on the outdated intranet page and for any suggestions for the redesign. This identified the following specific requests for documents which doctors were not able to access: falls proforma/guideline; hyponatraemia guideline; Parkinson's disease drug conversion to patch guideline.

2. A focus group was staged with the Foundation Year 2 doctors to discuss changes that might be made and any further suggestions/feedback. They asked for:

(a) Quick links to emergency guidance: adult life support protocol, seizures, hyperkalaemia, hypoglycaemia, chest pain, arrhythmias, asthma, anaphylaxis;

(b) A menu system with the following buttons: medicine guidelines, surgery guidelines, rotas, bleep list, medical calculators, incident reporting, feedback to the wards (could be anything from patient safety concern, to suggestion for improvement to positive feedback), common request forms (neurophysiology requests, ambulatory cardiac monitoring requests), routes of referral for specialities and services;

(c) Iron infusion guidelines; 
(d) A search bar.

1. The existing guidelines page was retrospectively evaluated in terms of the total number of mouse clicks required (19 clicks) and total time elapsed (140 seconds) to access a subset of five clinical guidelines (see Design section for full details).

\section{Design}

After gathering baseline information, we met with our hospital's Head of Clinical Audit \& Quality Improvement and a Clinical Audit \& Quality Improvement Assistant who was heavily involved in the technical aspects of website design. We discussed how the new page might function and explored possibilities for developing it as a resource for junior doctors beyond above and beyond being a guidelines page. Using the feedback already received, we collaborated on new version 1 of the intranet page.

To evaluate the performance of each iteration of the revised junior doctors' intranet site, we chose a varied subset of five different documents: 'Alcohol \& detox', 'Antibiotics'; 'Chest pain', 'Palliative care', and 'Trial without catheter (TWOC) referral'. These were chosen to represent a mix of commonly accessed guidelines, emergency guidelines and guidance which was less commonly accessed but perceived to be important to junior doctors.

Emulating similar projects [1,2], performance was measured in terms of:

1. Time (seconds) required to access a guideline;

2. Number of mouse clicks required to access a guideline; and

3. User satisfaction as reported through verbal feedback after testing.

We tested the 'old page', two draft versions of a new page, and then the finalised, live version of the Junior Doctor Guidelines page on the same three Foundation doctors at each iteration.

\section{Strategy}

PDSA cycles 1-4 took place over approximately six months, with most of the time between cycles being dedicated to website redesign.

PDSA cycle 1: 'old site'

We formally tested the pre-existing junior doctors' guidelines intranet page, sought feedback from all junior doctors via email and ran a focus group with Foundation Year 2 doctors.

Total time to access five guidelines: 140 seconds.

Total clicks to access five guidelines: 19 clicks.

Feedback: badly organised; antibiotic guidance accessed via the microbiology intranet site; TWOC referral was attempted to be found via the hospital intranet menu but users ended up opening up old emails to find the form which had been forwarded from another member of staff; palliative care guidance was accessed through the palliative care intranet menu.

Action: we collaborated on a new intranet site, responding to feedback.

PDSA cycle 2: new version 1

We formally evaluated the new junior doctors' guidelines intranet page (version 1) and invited immediate verbal feedback from testers.

Total time to access five guidelines: 34 seconds.

Total clicks to access five guidelines: 13 clicks.

Feedback: layout more logical; sparsity of surgical guidelines and other key areas of practice not covered; noted some coding glitches with the medicine subsection (flickering in/out of expanded lists).

Action: we collaborated on the next version of the intranet site, ensuring all available surgical guidelines were included and asking the coder to fix highlighted glitches.

PDSA cycle 3: new version 2

We formally evaluated the new junior doctors' guidelines intranet page (version 2), invited immediate verbal feedback from testers and sought feedback from the Foundation Programme team.

Total time to access five guidelines: 28 seconds.

Total clicks to access five guidelines: 12 clicks.

Feedback: requested to change section heading from 'palliative care' to 'palliative care \& elderly care' to group those documents more intuitively, asked to populate the 'emergency' menu with oneclick links to important guidance.

Action: we collaborated on the next version of the intranet site, modifying button titles and adding one-click access to emergency guidance.

PDSA cycle 4: new version 3 (live)

We launched the third iteration of the intranet site, and after four weeks bedding-in time, we formally tested the live junior doctors' guidelines intranet page and invited immediate verbal feedback from testers.

Total time to access five guidelines: 22 seconds.

Total clicks to access five guidelines: 12 clicks.

Feedback: re-feeding syndrome guidance link broken. 
Action: we submitted broken links to the coder and liaised with the Foundation Programme team to ensure that Foundation Programme representatives would be expected to maintain up to date guidance on the page as part of their job description.

\section{Results}

Intranet page performance was measured using the same three volunteers and the same five guidelines at each PDSA cycle in terms of:

1. Time (seconds) required to access a guideline;

2. Number of mouse clicks required to access a guideline; and

3. User satisfaction as reported through verbal feedback after testing.

Looking cumulatively at all five guidelines, we have shown a decrease in the number of clicks required access the sample of guidelines from 18 to 12 clicks. We have also demonstrated a corresponding decrease in the time required to access the sample of guidelines from 130 seconds to 22 seconds.

Taking each guideline individually, we have reduced the time taken to access all except 'Chest Pain', which has increased from 2 seconds to 4 seconds. This represents an insignificant change which we feel in itself will have no impact on patient safety; and we feel is outweighed by large gains in terms of accessing other guidelines. 'Alcohol \& Detox' reduced from 10 to 4 seconds; 'Antibiotics' from 8 to 4 seconds, 'Palliative Care' from 23 to 8 seconds, and 'TWOC referral; from 86 to 6 seconds.

Examining the mean number of clicks required per guideline, 'Alcohol \& Detox' increased from 1 to 2 clicks, 'Antibiotics' from 1.6 to 2 clicks, and 'Chest Pain' from 1.3 to 2 clicks. The increase in clicks is unlikely to affect patient safety as there was a decrease in time (except Chest Pain which we have already commented on). For 'Palliative Care' there was a decrease in the number of clicks from 4 to 3 clicks and for 'TWOC referral' from 10 to 2.6 clicks.

See supplementary file: ds6356.doc - "Results and intranet page demonstration \& graphical results"

\section{Lessons and limitations}

The page is not perfect. The visual appearance could be modernised and made more aesthetically pleasing. We have not been able to include everything that the junior doctors asked for: a feedback system for the wards has not been created and we could not include a search bar at this time due to coding issues. In addition to these finite issues, we recognise that ongoing work will be required to keep the intranet page up to date with new or updated documents. We have therefore ensured that responsibility for the electronic resource becomes a default responsibility of future Foundation Programme doctor representatives. Indeed, work on the intranet page is already underway by our successors. Our work has also raised awareness in non-junior doctor groups (e.g. specialities and allied healthcare professionals) of the page and we expect requests for the addition or update of documents will continue via the email links provided on the intranet page.

We have received no feedback to inform us that any existing document is missing from the intranet page. However, our work has highlighted a paucity of local guidance in certain areas (notably surgical specialities). We have therefore recommended that Foundation doctors are invited to participate in clinical guideline development.

\section{Conclusion}

We have achieved our aims: using qualitative methods (focus groups and feedback) and quantitive measures of guidelines access, we have implemented a Junior Doctor Guidelines page that is better accessed and has a more comprehensive coverage of the guidelines/documents which Foundation doctors need access to. We have created one-click access to emergency guidelines, clearly visible on the home page. We have organised the remaining guidelines according to broad speciality groupings, which has made them easy to find and is reflected in the decrease in time required to access them. In the sample we have tested, it is now possible to access any clinical guideline in less than 10 seconds. Finally and most importantly, we have increased user satisfaction: the junior doctors find the new page much easier to navigate and have commented on the usefulness of the added bleep list, aiding communication between doctors and services, and the 'referrals' section which has saved many people a great deal of time and effort identifying referral routes for services. Crucially, we have ensured that responsibility for the resource falls on future Foundation doctor representatives at our Trust so that it will continue to be maintained and developed.

\section{References}

1 Tarrant M, Honeyman C, Aquilina A, Young K. Improving the accessibility of trust guidelines for junior doctors at North Bristol NHS Trust. BMJ Qual Improv Report 2014;(3): doi:10.1136/bmjquality.u202211.w1101.

2 Robertson I, Smith A, Tucker J, Cilia E, Chen K, Marion R, Nesbitt J, Ramcharitar S, Greamspet MC. Improving accessibility of trust guidelines and protocols at the Great Western Hospital, Swindon. BMJ Qual Improv Report 2014;(3): doi:10.1136/bmjquality.u202615.w1585.

\section{Declaration of interests}

Nothing to declare.

\section{Acknowledgements}

We thank Calvin Turp and Frank Hamill for their work on realising the intranet page.

\section{Ethical approval}


BMJ Quality Improvement Reports

This work was a quality improvement project (registered under North Bristol NHS Trust ID Q1545) and the requirement for ethical approval was waived. 